\title{
FRACTURE OF THE LATERAL PROCESS OF THE TALUS
}

\author{
A Report of Thirteen Cases \\ Sadhan Kumar Mukherjee, Robert M. Pringle and Andrew D. Baxter,
Glasgow, Scotland \\ From the Orthopaedic and Accident Division, Glasgow Royal Infirmary, \\ and the Department of Anatomy, University of Glasgow
}

The lateral process of the talus is the most lateral part of the body of that bone and extends from the lower margin of the vertical articular surface for the fibula to the posterior inferior surface of the talus (Fig. 1). It has articular and non-articular surfaces. Its articular surface is the most lateral part of the posterior inferior articular surface of the talus. A fracture of the process therefore involves the posterior subtalar joint-it is not an "ankle fracture" in the ordinary sense of that term.

Marottoli (1942) collected six reported cases of this fracture and added four of his own. There have been only a few reports in the English literature. Bonnin (1950) mentioned the fracture, which he said could be treated conservatively because it was usually undisplaced. Milch and Milch (1959) described a fracture in this region caused by excessive eversion of the foot, which would appear to have been a fracture of the lateral process of the talus. Dimon (1961) reported three displaced fractures and pointed out that they could lead to considerable disability. Since then a few articles have been published (Cimmino 1963, Hawkins 1965, Fjeldborg 1968). Hawkins had the largest series, thirteen cases collected from fifty consecutive talar fractures, and regarded this as the second most common fracture of the talus.

The injury is thought to be uncommon.

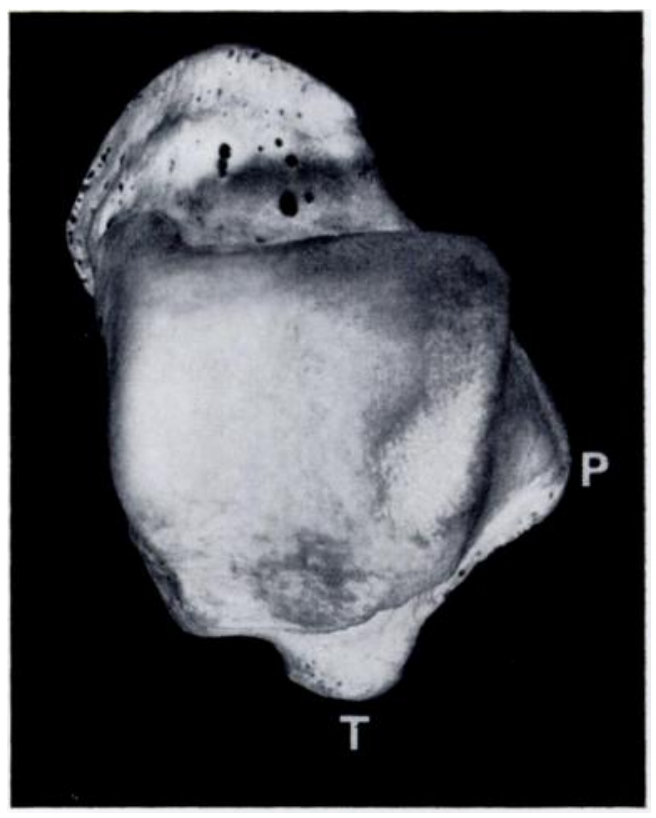

Fig. 1

A dorsal view of a right talus showing the lateral process (P) and the trigonal tubercle (T). The significance of the radiographic appearance is not always appreciated and definitive treatment is often delayed. For these reasons we think it is worth while reporting thirteen cases collected over a period of thirteen months from approximately 1,500 cases of sprain and fracture of the ankle region, an incidence of 0.86 per cent.

\section{ANATOMICAL STUDIES}

The purposes of the anatomical studies were firstly, to define the lateral process of the talus as above; secondly, to determine its internal structure; and thirdly, to study the ligaments of this area and their functional significance in relation to movements of the ankle and subtalar joints.

The superior facet of the talus widens anteriorly. It fits into a tapered mortice bound together anteriorly and posteriorly by the inferior tibio-fibular ligaments, which run infero-laterally. This is said to allow some separation of the malleoli under stress. On dorsiflexion of the ankle the talus is firmly wedged into the mortice of the ankle. 
The ligamentous structures supporting the ankle joint laterally are the three parts of the lateral collateral ligament, namely, the anterior and posterior talo-fibular ligaments and the calcaneo-fibular ligament (Fig. 2). From studies of specially taken radiographs it seems that the posterior talo-fibular ligament is taut on dorsiflexion whereas the anterior ligament is slack; the converse applies on plantarflexion (Figs. 3 and 4). The calcaneo-fibular ligament, which runs postero-inferiorly at about 45 degrees, is tense on dorsiflexion. The nearly horizontal disposition of the talo-fibular ligaments

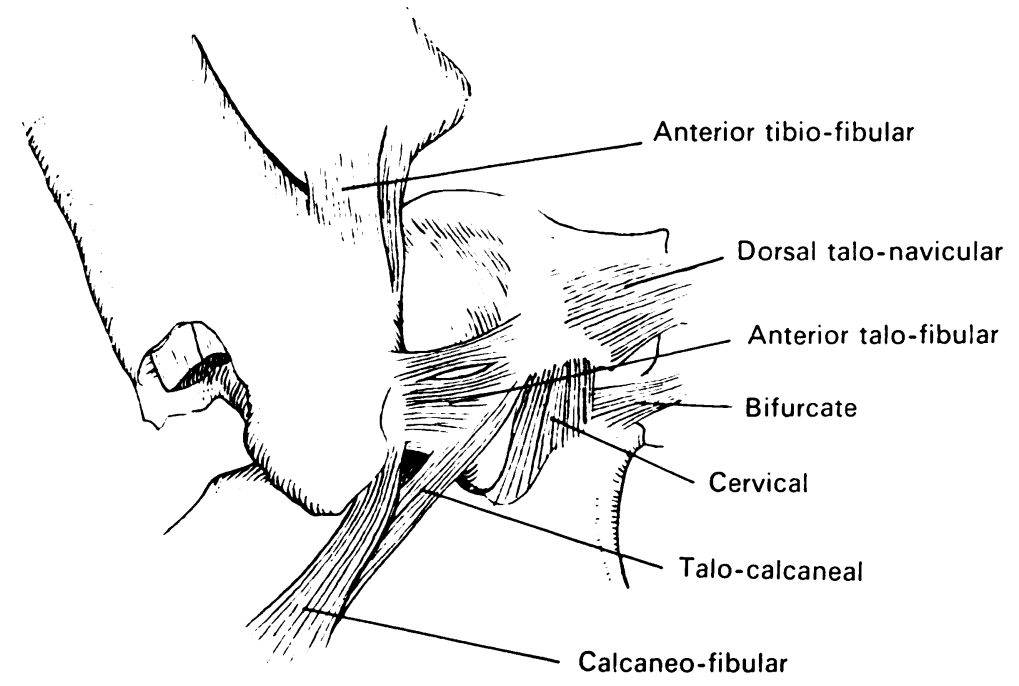

Fig. 2

A drawing of a dissection of the ligaments related to the lateral aspect of the talus.

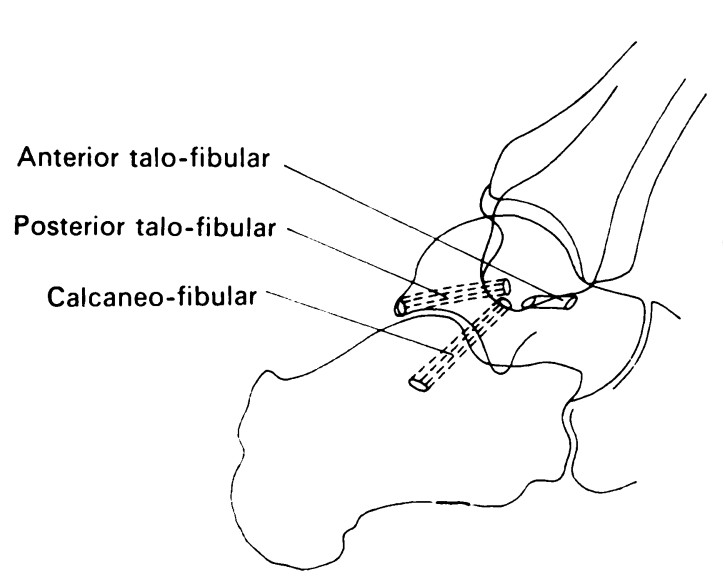

Fig. 3

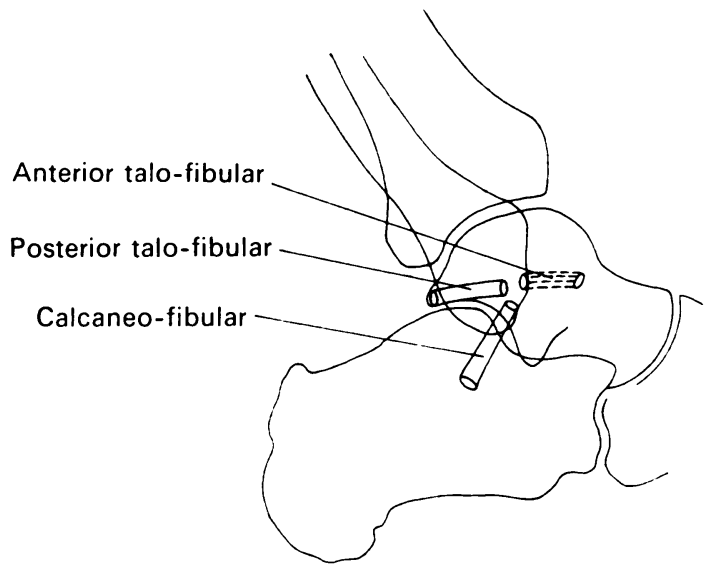

Fig. 4

Two drawings made from tracings of radiographs taken along the axis of the dome of the talus to show the relative lengths and stresses in the anterior and posterior talo-fibular and calcaneo-fibular ligaments in dorsiflexion (Fig. 3) and in plantar-flexion (Fig. 4). Ligaments shown with interrupted lines are under tension.

suggests that they take little part in limiting inversion of the ankle joint, but rather limit forward and backward gliding of the leg bones upon the talus.

Lying deep to and parallel with, but a little anterior to the calcaneo-fibular ligament is the lateral talo-calcaneal ligament (Fig. 2). This is a fairly small band and its action is to restrict separation of the surfaces of the talo-calcaneal joint. It is joined in this action by the calcaneo-fibular ligament, which acts over both ankle and subtalar joints. Inversion and eversion of the foot take place at both the subtalar and the midtarsal joints. It can be seen that dorsiflexion of the ankle with resultant tension in the calcaneo-fibular ligament will reduce movement of the subtalar joint, and this can be 


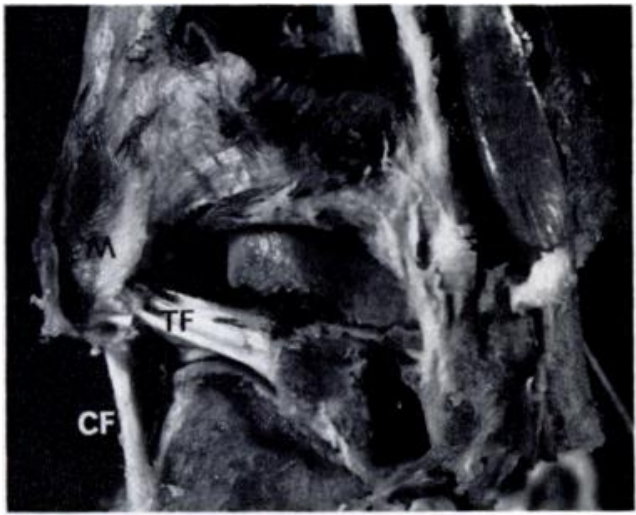

Fig. 5

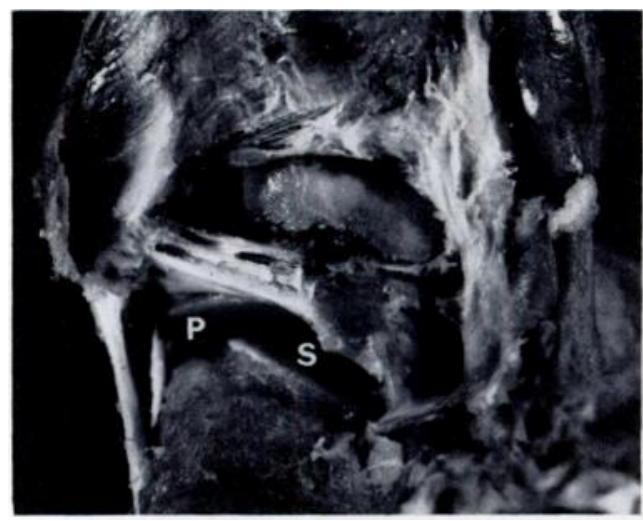

Fig. 6

Figure 5-A photograph of a dissection showing the posterior aspect of the left ankle in the neutral position The lateral malleolus (M), the calcaneo-fibular ligament (CF), and the posterior talo-fibular ligament (TF) with the subtalar joint just below it are all clearly seen. Figure 6-The same dissection as in Figure 5 but with the foot in inversion. The subtalar joint $(\mathrm{S})$ has opened. The lateral process of the talus $(\mathrm{P})$ is now much more clearly visible.

observed on passive movement of the living foot. The cervical ligament lies well to the lateral side of the axis of the subtalar movement and its function is therefore to limit inversion of the foot at the subtalar joint.

In the normal position of the foot there is close apposition between the curved superior facets of the calcaneus and the corresponding talar facets (Fig. 5). This is not the case in inversion, when the subtalar joint is opened up (Huson 1961). In a dissected specimen it can be seen that on inversion

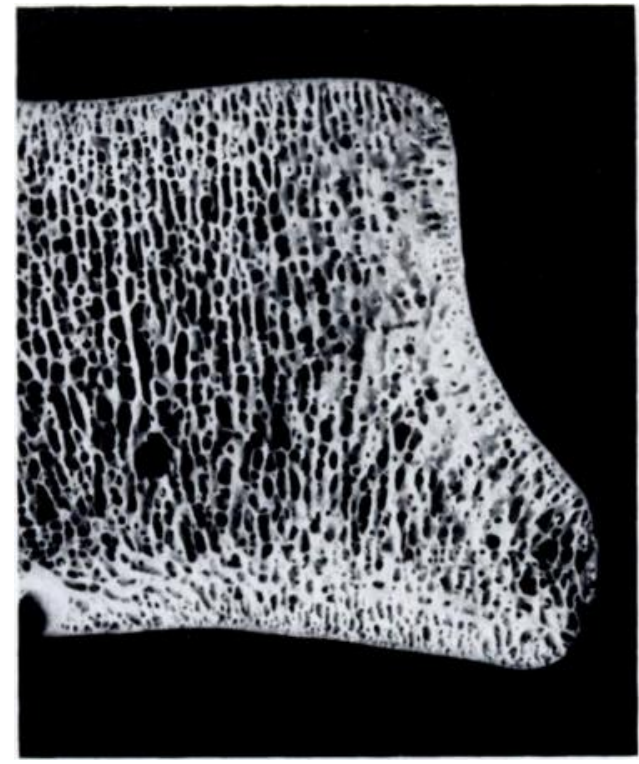

Fig. 7

A ground section of the talus through the lateral process showing the trabecular structure.

the lateral part of the posterior talar facet rides up on to the posterior calcaneal facet, causing separation of large areas of these facets (Fig. 6). Under these circumstances the area of the lateral process is called upon to transmit compression forces between the tibia and the calcaneus. On examination of the trabeculation of the talus it is clear that adequate provision is made for the transmission of such forces between the supero-lateral and inferior surfaces near the root of the lateral process. There is, however, little trabeculation to be seen at the apex (Fig. 7).

VOL. 56 B, NO. 2, MAY 1974 


\section{MECHANISM OF INJURY}

Dimon (1961) postulated that the mechanism causing a fracture of the lateral process of the talus is compression caused by dorsiflexion and slight lateral rotation of the foot.

However, Hawkins (1965) based his opinion on

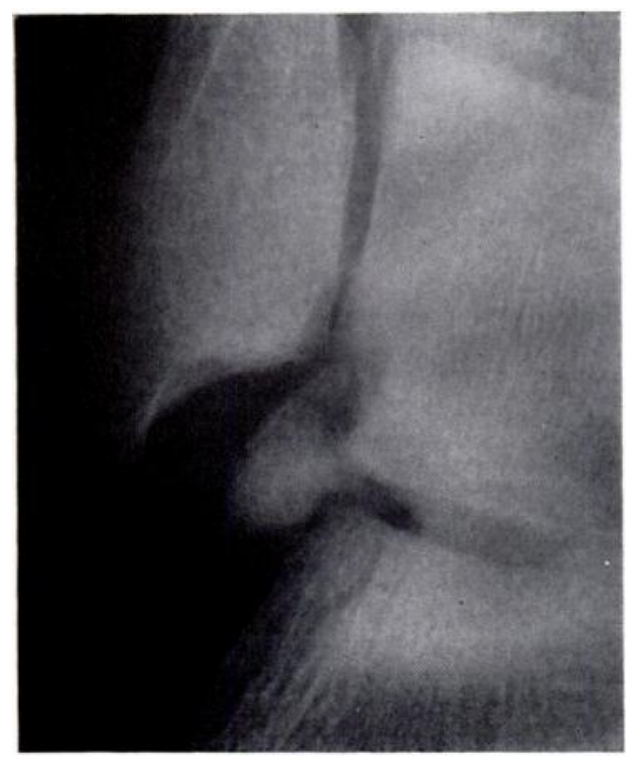

FIG. 8

Case $1-A$ radiograph taken with the ankle at $\mathbf{0}$ degrees and the leg rotated inwards 20 degrees showing a fracture of the lateral process of the talus and the amount of displacement. the work of Huson (1961) and suggested that the fracture occurs from forced dorsiflexion of the foot with associated inversion. According to Fjeldborg (1968) this fracture may arise from a fall on to the dorsally flexed, supinated foot, which is similar to the mechanism advanced by Hawkins. We were able to reproduce this type of fracture by a compression force applied to the dome of the talus with the talo-calcaneal joint in inversion.

We have also studied thirteen patients with fractures of the lateral process of the talus seen between November 1971 and December 1972. Eight of these patients came to us soon after the injury. We were able to establish that all the patients had sustained an inversion injury and that seven of the thirteen also had dorsiflexion of the foot at the time of injury. From our anatomical and clinical studies we agree with the opinions of Hawkins and of Fjeldborg that this fracture occurs from compression of the lateral process of the talus with the foot in inversion and dorsiflexion.

\section{CASE REPORTS}

Case 1-A man aged thirty injured his right ankle by jumping down four steps of a flight of stairs. On examination ten days later on November 10,1971, the ankle region was swollen, especially on the lateral side, and was acutely tender over the antero-lateral aspect. Radiographs showed a displaced fracture of the lateral process of the talus (Fig. 8). At operation the next day the fracture was found to involve a considerable part of the articular surface of the postero-lateral component of the talo-calcaneal joint; it was comminuted, unstable and displaced. The fragments were removed. Weight-bearing was avoided for five weeks. When last reviewed fifteen months after the injury he was completely free from symptoms and had a full range of ankle and tarsal movements. The radiographs showed no further change.

Case 2-A man aged fifty-four sustained an inversion injury to the left ankle in a fall from a height. On examination the next day there was moderate swelling of the antero-lateral aspect of the ankle and also over the medial malleolus. Tenderness was most marked over the lateral part of the talus. Radiographs showed a fracture of the lateral process (Fig. 9). At operation the fragment was found to be displaced but still attached to the fibula by the anterior talo-fibular ligament and partly attached to the main body of the talus. The fragment, which measured approximately $18 \times 6 \times 5$ millimetres, was reduced and held in position by two Kirschner wires (Figs. 10 and 11). A below-

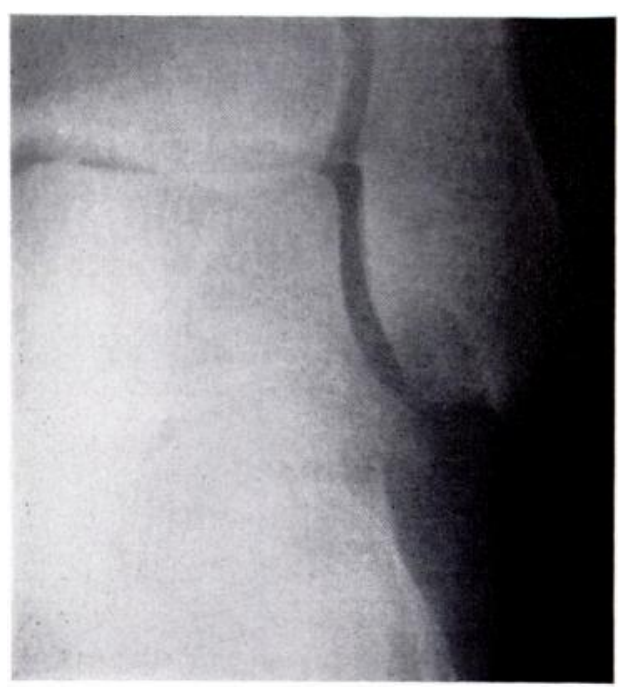

FIG. 9

Case 2-A radiograph showing a fracture of the lateral process of the talus.

THE JOURNAL OF BONE AND JOINT SURGERY 


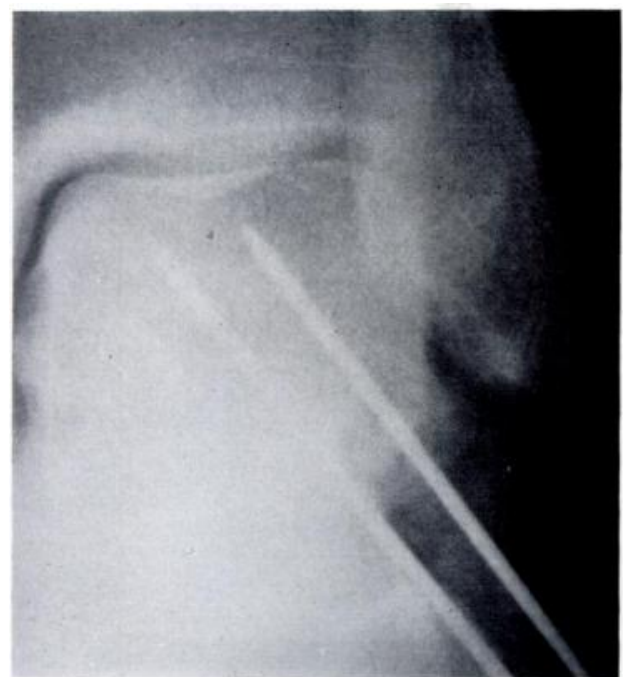

Fig. 10

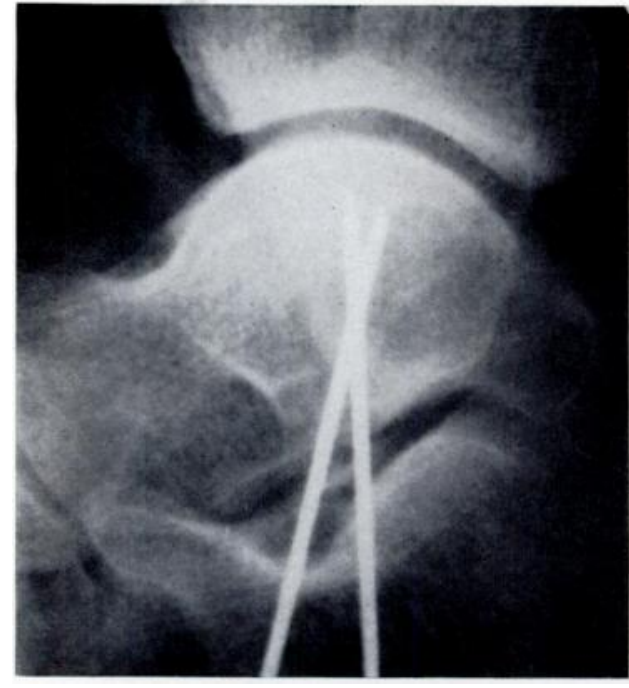

Fig. 11

Case 2-Antero-posterior and lateral radiographs showing the reduced fracture held by two Kirschner wires.

knee plaster cast was worn for six weeks, after which the wires were removed and the ankle mobilised. Ten months after the injury the patient's only complaint was of slight instability of the ankle on uneven ground and occasional vague discomfort over the antero-lateral aspect. Radiographs showed the fracture united (Fig. 12). When last seen fourteen months after the injury he was completely free from symptoms and had a full range of movement.

Case 3-A man aged twenty sustained an inversion and dorsiflexion injury to his right ankle from a fall. A radiograph taken on December 23, 1971, revealed a fracture of the lateral process of the talus (Fig. 13). At operation a month later a large loose fragment was removed and the defect in the talus was clearly seen. Ten months after the injury he was free of symptoms and had an unrestricted range of ankle and foot movements. Radiographs showed no further changes in the subtalar joint. Fourteen months after the injury the findings were the same.

Case 4-A woman aged forty-two was first seen at another hospital on December 24, 1971, with a history of a recent inversion injury to the left ankle. Radiographs showed a fracture of the lateral process of the talus (Fig. 14). She was later referred to us and at operation on January 19, 1972, a fracture with gross displacement was found. The fragment, which measured $10 \times 8 \times 4$ millimetres, was removed and a below-knee plaster cast applied. This was retained for four weeks and weight-bearing was avoided for six weeks altogether. When last seen ten months after the injury she had no complaints. Plantarflexion, dorsiflexion and inversion of the foot were full but eversion was slightly restricted. Radiographs

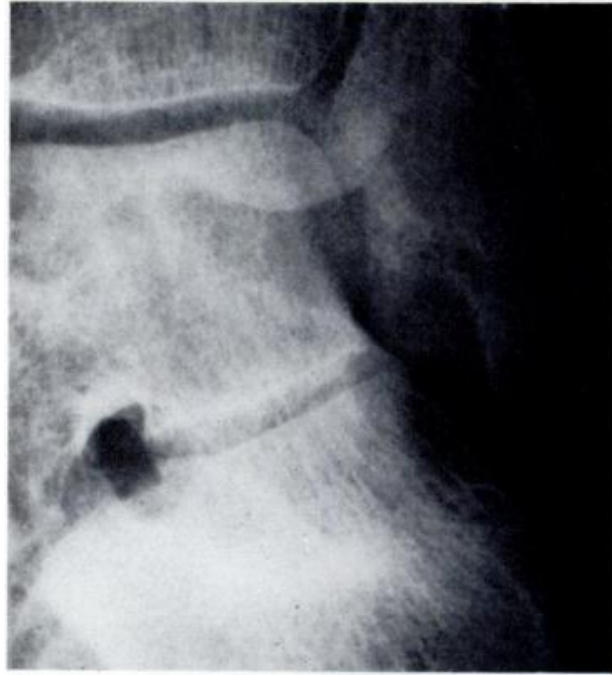

FIG. 12

Case 2-A radiograph taken ten months after injury showing union of the fracture in apparently normal alignment. showed no further change.

Case 5-A woman aged fifty-three was first seen on May 1, 1972, with a history of an inversion and compression injury to the left ankle joint the previous day. There was moderate swelling and tenderness over the antero-lateral aspect. Radiographs showed a fracture of the lateral process of the talus. At operation a comminuted fracture with displacement was found and the fragments were removed. A below-knee plaster cast was retained for three weeks and weight-bearing was avoided for six weeks. 
Five months after operation she was complaining of slight discomfort in the ankle at the end of the day and felt some uncertainty in walking on uneven ground, but she could run without discomfort. Radiographs showed normal appearances in the ankle and subtalar joints. Nine months after the injury there were no symptoms and movements were full.

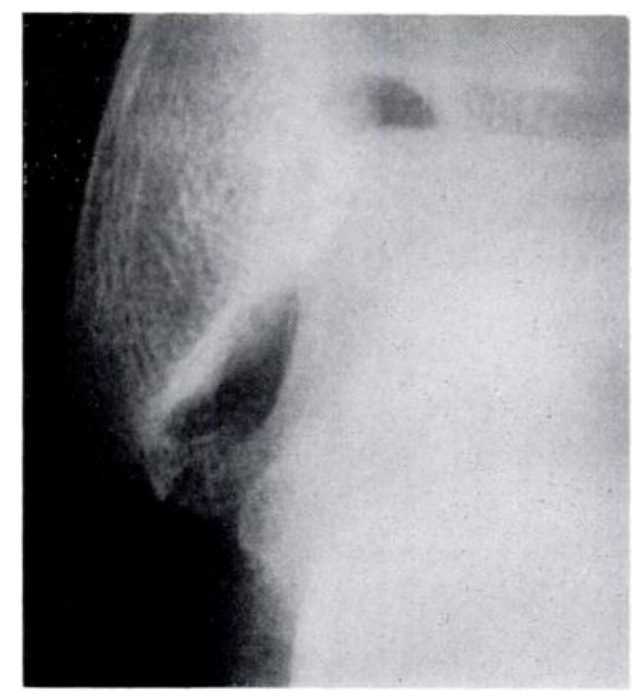

FIG. 13

Case 3-A radiograph showing a displaced fracture of the lateral process of the talus.

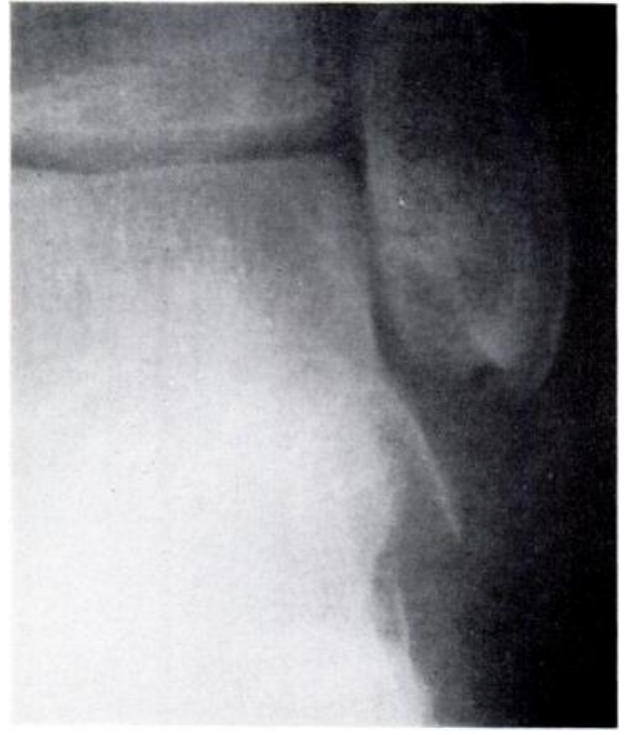

FIG. 14

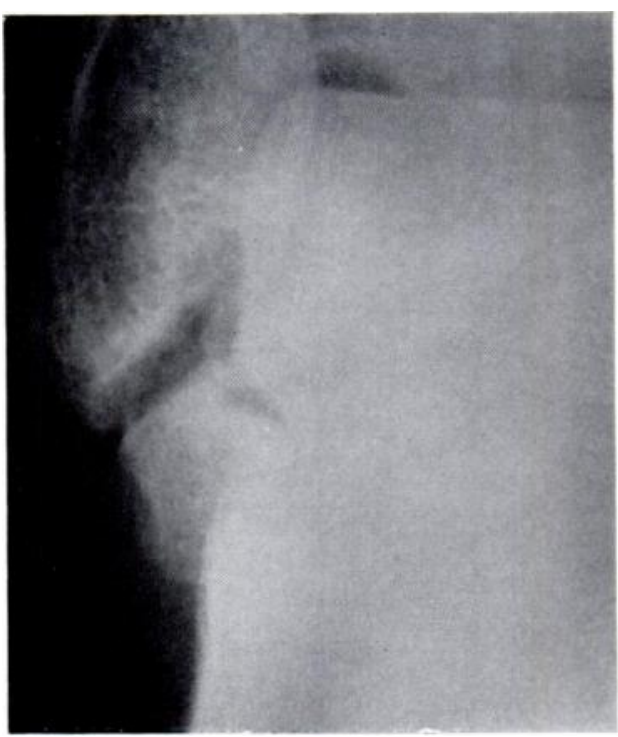

Fig. 15

Figure $14-$ Case 4. A radiograph showing a fracture of the lateral process of the talus. Figure 15Case 6. A radiograph taken three years after injury showing non-union of the fracture and local degenerative changes.

Case 6-A man aged forty-nine was first seen on July 9, 1969, for injuries to the right ankle and elbow sustained in a fall from a height of three metres (ten feet). A comminuted fracture of the head of the radius was treated by operation. The right ankle was swollen and tender over the antero-lateral aspect. Radiographs on that date showed a fracture of the lateral process of the talus the significance 
of which was not appreciated, and it was treated as a case of fracture of the lateral malleolus. He recovered well from the elbow injury, but had persistent symptoms of pain, instability and stiffness of the ankle and foot. Three years after the injury the symptoms had worsened. Radiographs now showed an ununited fracture of the lateral process with degenerative changes (Fig. 15), but the patient refused operative treatment.

Case 7-A man aged thirty-five attended on April 6, 1972, for a twisting injury of the left ankle sustained in a fall from a height of three and a half metres (twelve feet). There was swelling on both sides of the ankle joint with tenderness mainly over the antero-lateral aspect. Radiographs showed a fracture of the lateral process of the talus with slight displacement. He was advised operative treatment but decided against it because his home was over 300 miles away. A below-knee plaster cast was applied and he was referred to his general practitioner. Repeated attempts to contact the patient have been unsuccessful.

Case 8-A man aged sixty-eight attended on April 28, 1972, soon after being knocked down by a car which ran over his left foot. The left ankle was bruised and swollen with tenderness below both malleoli. Radiographs revealed a displaced fracture of the lateral process of the left talus (Fig. 16),

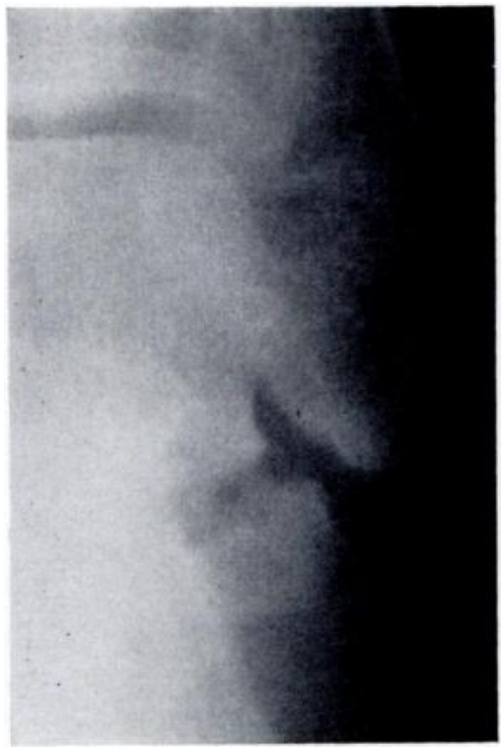

FIG. 16

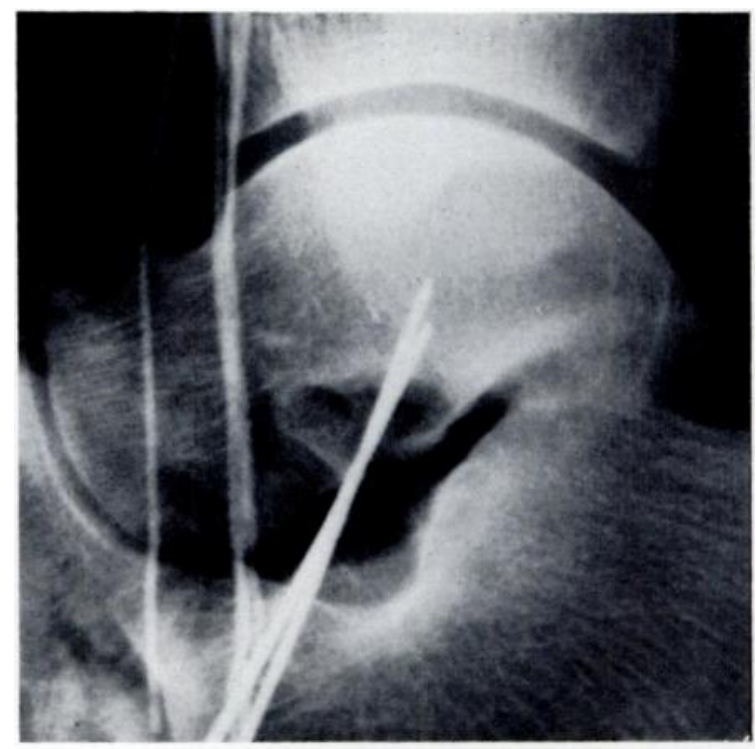

FiG. 17

Case 8. Figure 16-A radiograph showing the fracture of the lateral process of talus. Figure 17-A lateral radiograph showing the fracture held reduced by two Kirschner wires.

and also a fracture of the cuboid. At operation the same day a large fragment of the lateral process was found detached. Small pieces of crushed bone around the large fragment were excised and the main fragment was held reduced with two Kirschner wires (Fig. 17). A below-knee plaster cast was applied. Weight-bearing was avoided for four weeks, after which the wires were removed, apart from a piece which broke off in the talus. Seven months after the accident he complained of slight stiffness of the foot and ankle, all movements of which were slightly restricted. There was still some tenderness over the tip of the lateral malleolus, with swelling of the ankle and foot. Radiographs showed the fracture united but a moderate degree of local osteoporosis. Nine months after injury symptoms were minimal and the range of ankle and foot movements had improved.

Case 9-A man aged twenty attended another hospital on March 28, 1971, after a traffic accident in which both ankles were injured by acute dorsiflexion of both feet on the brake and clutch pedals. He was treated by below-knee plaster casts for four months, followed by physiotherapy.

When seen at Glasgow Royal Infirmary on December 29, 1971, he complained of pain on the lateral side of the right ankle, especially when walking on uneven ground. All movements of the foot and ankle were restricted, with marked tenderness over the lateral malleolus and immediately anterior. Radiographs showed an old fracture of the lateral process of the right talus with degenerative change in the subtalar joint, and a detached fragment of lower end of fibula. The original radiograph clearly showed the talar fracture (Fig. 18). 
At operation on January 28, 1972, a large piece of fibular bone was excised, the peroneal tendons were freed and osteophytes and bone fragments arising from the lateral process of the talus were removed. The subtalar joint on the lateral side was found to be arthritic. Full weight-bearing was avoided for three weeks. Seven months after operation the patient had some pain over the lateral aspect of the ankle but was able to work as a motor mechanic. Movements of the ankle were full

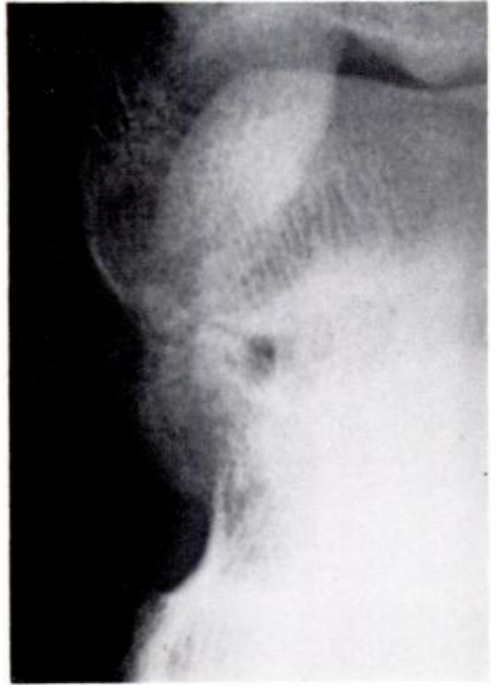

FIG. 18

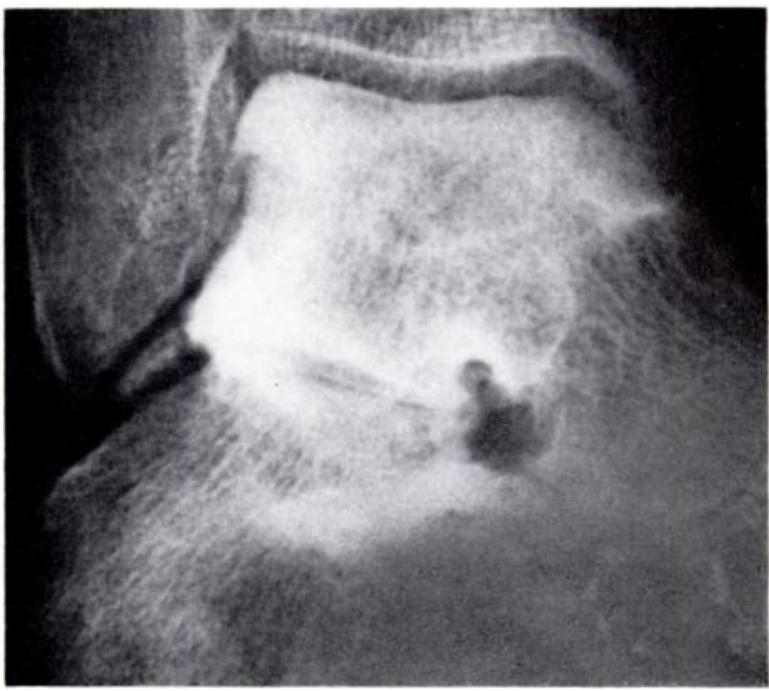

FIG. 19

Case 9. Figure 18-The original radiograph showing a fracture of the lateral process of the talus.

Figure 19-A radiograph taken nineteen months after injury showing some new bone formation and advanced degenerative change in the subtalar joint.

but the subtalar joint was stiff. A radiograph showed some new bone formation at the site of fracture and degenerative changes in the subtalar joint (Fig. 19). When last seen twenty-two months after the injury the symptoms were sufficient to warrant subtalar fusion, which advice the patient has accepted.

Case 10-A man aged forty-two had his left forefoot crushed in a hydraulic ram on August 14, 1971, sustaining compound fractures of the great, second, third and fourth toes which were treated by terminal amputations. Healing was slow but he was eventually discharged from out-patient attendance on December 1, 1971. On June 26, 1972, the patient was seen again at the request of his general practitioner because of persistent pain in the left ankle. There was discomfort on palpation just below the lateral malleolus and ankle movements were slightly restricted. A radiograph showed an old fracture of the lateral process of the talus.

At operation on July 28,1972 , the fragment was found to have united with slight deformity. Small loose cartilaginous fragments at the site of the injury were removed. After operation the patient avoided weight-bearing by the use of crutches for three weeks. Eighteen months after the injury he had minimal discomfort, but movements of the ankle and foot were free and radiographs showed no further change.

Case 11-A man aged thirty-five fell from a height of three metres (ten feet) on November 10, 1972, and injured his left ankle. The mechanism appeared to be inversion and dorsiflexion. Clinically there were abrasions and swelling over the lateral aspect, with tenderness maximal over the lateral part of talus just below the tip of the malleolus. Radiographs showed a fracture of the lateral process of the talus.

Initially he was treated as a case of fracture of the lateral malleolus by immobilisation in a plaster cast, but exploration was carried out on December 1, 1972, after the correct diagnosis had been made. A comminuted fracture of the lateral process with involvement of the subtalar joint was found and the loose fragments were removed. After operation he was treated by immobilisation in a plaster cast for two weeks and no weight-bearing for a further two weeks. This patient was re-admitted on August 6. 1973, for excision of a painful scar following the exploration of the ankle. It was noted that movement of the subtalar joint was full and painless. 
Case 12-A woman aged twenty-four sustained an inversion injury of the left ankle on December 7, 1972, and was treated by immobilisation in a plaster cast. On removal of the cast two weeks later, bruising and swelling of the lateral side of the ankle and foot were found and there was acute tenderness over the lateral process of the talus. A radiograph showed a fracture of the lateral process of the talus.

At operation on December 21,1972 , a fragment of talus measuring $6 \times 8$ millimetres was found lying below the tip of the fibula and was removed. A below-knee plaster cast was applied and weightbearing was avoided for four weeks. This patient was last seen on March 16, 1973, when she was symptom-free and had a full range of movement of the ankle and subtalar joints. Radiographs of the subtalar joint were normal.

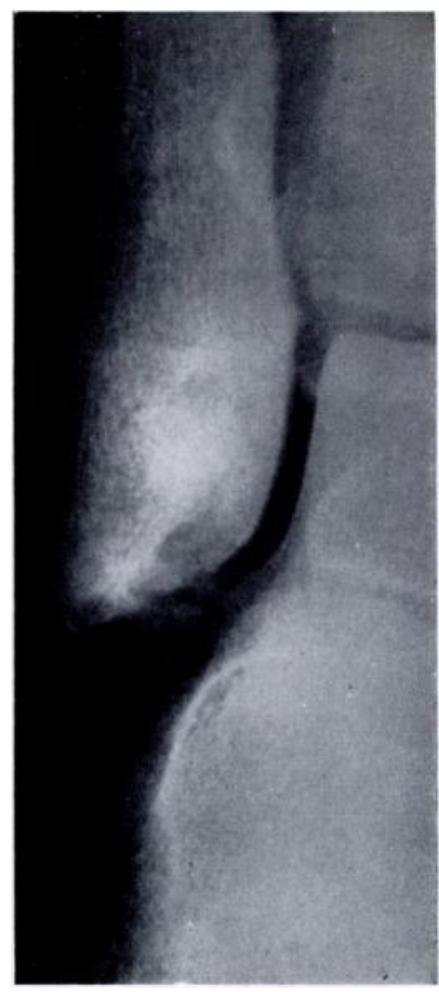

FIG. 20

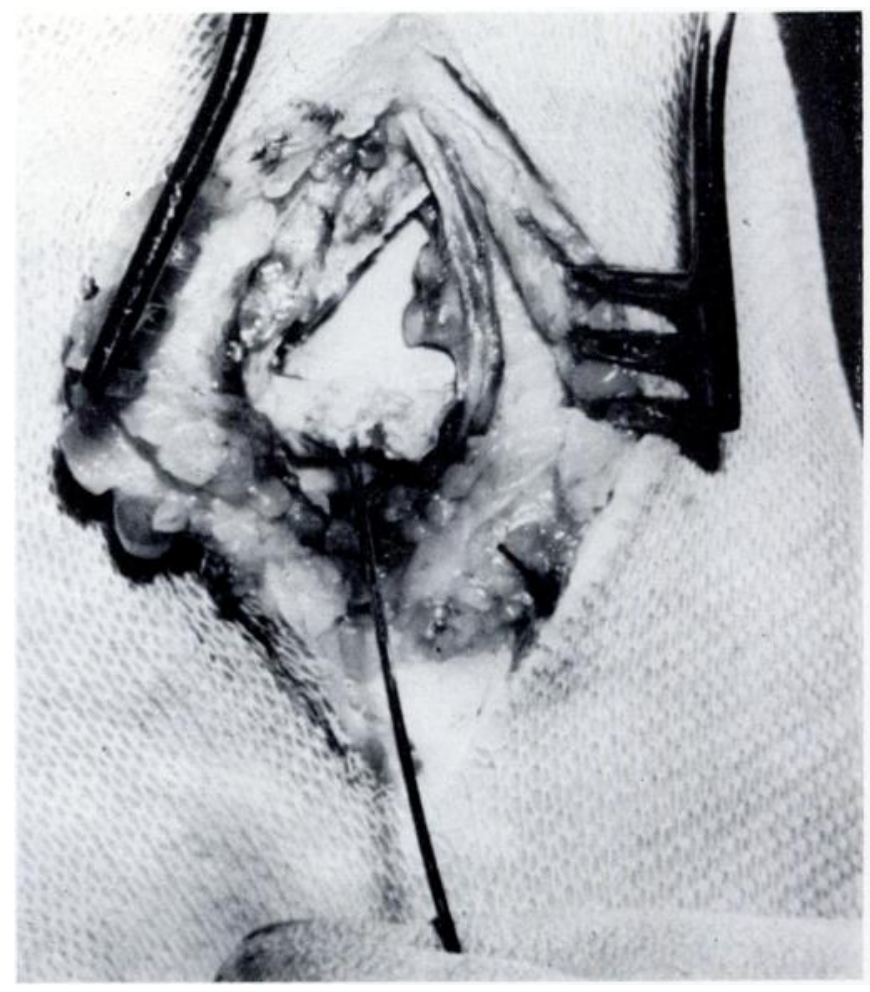

Fig. 21

Case 13. Figure 20-A radiograph showing a fracture of the lateral process of the right talus. Figure 21-A photograph taken at operation. The right foot is in full equinus; the incision is vertical; the large facet on the talus for articulation with the fibula is well shown. Immediately below this the site of fracture of the lateral process can be seen, with some new bone formation over it. The point of the thin probe is in the subtalar joint.

Case 13-A woman aged fifty-four sustained an inversion injury of the right ankle on August 30, 1972. When first seen by us on November 8, 1972, she was diagnosed as a case of sprained ankle and treated in a below-knee walking cast. Radiographs taken on December 6, 1972, three months after the injury, showed a fracture of the lateral process of the right talus (Fig. 20). At this time the patient had increased pain in the right ankle. At operation on January 12, 1973, a small loose osteochondral fragment of lateral process was found, together with some irregularity and new bone formation over the non-articular part of the lateral process (Fig. 21). The subtalar joint appeared normal. The loose fragments were removed and the ankle immobilised in a below-knee plaster cast. This patient was last seen on August 9, 1973, when she was symptom-free and had a full range of movement of the ankle and subtalar joints.

\section{DISCUSSION}

Our experience suggests that, contrary to the general view, this fracture is relatively common. Although the clinical picture may be masked by soft-tissue injury, a helpful sign is acute local tenderness over the lateral process of the talus, just below the tip of the lateral 
malleolus. The fracture involves the lateral part of the articular surface of the posteroinferior part of the talus, and in our experience the fragment is usually comminuted or displaced. An irregularity or the presence of a loose body may lead to arthritic changes in the subtalar joint. Thus, of the thirteen cases reported by Hawkins, three had developed within six to twenty-four months from injury degenerative changes which warranted subtalar fusion, but all three had had inadequate treatment.

It appears from the study of our thirteen cases that early treatment produces good results (Table I), though it is much too soon to come to a final conclusion. One of our patients (Case 7) has been lost to follow-up, and the most recent (Cases 11 to 13) are still under

TABLE I

analysis of Thirteen Cases of Fracture of the Lateral Process of the Talus

\begin{tabular}{|c|c|c|c|c|c|c|}
\hline $\begin{array}{c}\text { Case } \\
\text { number }\end{array}$ & $\begin{array}{c}\text { Age } \\
\text { (years) }\end{array}$ & Sex & $\begin{array}{l}\text { Time from } \\
\text { injury to } \\
\text { operation }\end{array}$ & Treatment & $\begin{array}{c}\text { Follow-up } \\
\text { (months) }\end{array}$ & Results \\
\hline 1 & 30 & Male & 1 day & Excision & 15 & Symptom free \\
\hline 2 & 54 & Male & 1 day & Replacement & 14 & Symptom free \\
\hline 3 & 20 & Male & 1 month & Excision & 14 & Symptom free \\
\hline 4 & 42 & Female & 1 month & Excision & 10 & Symptom free \\
\hline 5 & 53 & Female & 1 day & Excision & 9 & Symptom free \\
\hline 6 & 49 & Male & $*$ & Conservative & 35 & $\begin{array}{l}\text { Subtalar pain, } \\
\text { advised fusion }\end{array}$ \\
\hline 7 & 35 & Male & $*$ & $\begin{array}{c}\text { Initially } \\
\text { conservative }\end{array}$ & * & * \\
\hline 8 & 68 & Male & Same day & Replacement & 9 & Discomfort only \\
\hline 9 & 20 & Male & 9 months & $\begin{array}{l}\text { Excision and } \\
\text { cheilotomy }\end{array}$ & 22 & $\begin{array}{l}\text { Awaiting } \\
\text { subtalar fusion }\end{array}$ \\
\hline 10 & 42 & Male & 10 months & Exploration & 18 & Discomfort only \\
\hline 11 & 35 & Male & 3 weeks & Excision & 9 & $\begin{array}{l}\text { Under treatment } \\
\text { for painful scar }\end{array}$ \\
\hline 12 & 24 & Female & 2 weeks & Excision & 4 & Symptom free \\
\hline 13 & 54 & Female & 4 months & Excision & 12 & Symptom free \\
\hline
\end{tabular}

* Indicates "details not known".

treatment, so that no useful comment can be made on their progress. This leaves nine patients for review. The first five have no symptoms nine to fifteen months after operation performed on three within twenty-four hours and on the other two within a month of injury. Two patients have had poor results; they were diagnosed six months after injury (Case 6) and four months (Case 9).

A large proportion of these fractures must go unrecognised. In the light of our experience, however, we believe that a large single fragment should be reduced accurately by open operation in order to ensure congruity of the surfaces of the subtalar joint, and that small or comminuted fragments are best treated by removal, in either case soon after the injury.

\section{SUMMARY}

1. Thirteen cases of fracture of the lateral process of the talus seen over a period of thirteen months are reported. 
2. The mechanism of the injury would appear to be inversion strain of the foot with dorsiflexion of the ankle.

3. The diagnosis would no doubt be made more often if the possibility of this fracture were kept in mind, and if radiographs of good quality were taken with the ankle at 0 degree and the leg rotated inwards 10 to 20 degrees.

4. With regard to treatment, early operation appears to give good results, a single large fragment being reduced accurately and small or comminuted fragments being removed.

We would like to thank Mr J. White, Mr J. H. Miller, Mr A. B. Young, Consultant Orthopaedic Surgeons, Glasgow Royal Infirmary, for allowing us to report their cases. Our thanks also go to Mrs D. Kelly, Department of Medical Illustration, Glasgow Royal Infirmary, and to the medical artists of the Anatomy Department, University of Glasgow, for the illustrations.

\section{REFERENCES}

Bonnin, J. G. (1950): Injuries to the Ankle. New York: Grune and Stratton.

Cimmino, C. V. (1963): Fracture of the lateral process of the talus. American Journal of Roentgenology, Radium Therapy and Nuclear Medicine, 90, 1277-1280.

Dimon, J. H. (1961): Isolated displaced fracture of the posterior facet of the talus. Journal of Bone and Joint Surgery, 43-A, 275-281.

FJELDBORG, O. (1968): Fracture of the lateral process of the talus. Acta orthopaedica Scandinavica, 39, 407-412.

Hawkins, L. G. (1965): Fracture of the lateral process of the talus. Journal of Bone and Joint Surgery, 47-A, $1170-1175$.

Huson, A. (1961): Een ontleedkundig-functioneel Onderzoek van de Voetwortel. (An Anatomical and Functional Study of the Tarsal Joints.) Leiden: Drukkerij Luctor et Emergo.

Marotroli, O. R. (1942): Sobre las fracturas de la apófisis externa del astrágalo. Anales de Cirugia, 8, 58. Cited in current literature. Journal of Bone and Joint Surgery, 1943, 25, 225.

Milch, H., and MiLch, R. A. (1959): Fracture Surgery, p. 452. New York: Paul B. Hoeber. 